\title{
UTILIZAÇÃO DE LODO DE UMA FÁBRICA DE PAPEL PARA SORÇÃO DE COBRE, CÁDMIO E CROMO DE SOLUÇÕES AQUOSAS
}

\author{
D. M. VENEU ${ }^{1,2,3}$ \\ A. O. IZOLANI ${ }^{2,3}$ \\ B. B. BARBOSA ${ }^{2}$ \\ D. OLIVEIRA ${ }^{2}$, C. \\ C. R. MAUAD ${ }^{4,3}$, \\ ${ }^{1}$ Universidade Federal do Rio de Janeiro/Escola de Química \\ ${ }^{2}$ Universidade Severino Sombra/CECETEN \\ ${ }^{3}$ Centro Universitário Geraldo Di Biase/ICETE \\ ${ }^{4}$ Pontifícia Universidade Católica do Rio de Janeiro/Departamento de Química \\ ${ }^{5}$ Empresa Brasileira de Pesquisa Agropecuária/Departamento de Fertilidade do Solo \\ E-mail para contato: diegomveneu@ yahoo.com.br
}

D. V. B. CAMPOS ${ }^{5,2}$,

\begin{abstract}
RESUMO - Neste trabalho, foi avaliado o potencial do lodo proveniente de um decantador primário de um sistema de tratamento de efluentes de lodos ativados em uma indústria do segmento de celulose e papel como sorvente para a remoção de $\mathrm{Cu}$, $\mathrm{Cd}$ e $\mathrm{Cr}$ de soluções aquosas. A capacidade de remoção dos íons $\mathrm{Cu}, \mathrm{Cd}$ e $\mathrm{Cr}$ pelo lodo é fortemente influenciada pelo $\mathrm{pH}$ do meio, obtendo-se a maior eficiência em $\mathrm{pH} 5,0$ para o $\mathrm{Cu}$ e $\mathrm{Cd}$, correspondendo a $69,4 \%$ e $96,4 \%$, respectivamente, e $\mathrm{pH} 4,0$ para o $\mathrm{Cr}$, correspondendo a 99,9\%. As remoções de $\mathrm{Cu}, \mathrm{Cd}$ e $\mathrm{Cr}$ sofreram uma relevante influência pela concentração de lodo, sendo os melhores resultados obtidos com 3,0 $\mathrm{g} / \mathrm{L}$, correspondendo a 35,2\%, 99,9\% e 99,8\%, respectivamente. As interações entre os íons metálicos e os grupos funcionais da biomassa foram confirmadas pelas análises de IV-FT e MEV/EDS. Os grupos funcionais envolvidos na sorção das espécies metálicas incluíram os grupos carboxila, amina, mercaptanos e hidroxila.
\end{abstract}

\section{INTRODUÇÃO}

O Brasil destaca-se mundialmente por produzir e abastecer os mercados com expressivos volumes de papel de embalagem, papéis de imprimir e escrever e papel cartão. Nos últimos dez anos, o País aumentou sua produção em 27,0\%, com crescimento médio de 2,7\% ao ano, em 2012, foram produzidas 10,3 milhões de toneladas do produto (Bracelpa, 2014). Para suprir toda essa demanda, dois recursos naturais são extremamente importantes, um deles está relacionado diretamente ao produto que é a celulose, o outro é a água. Embora vários avanços tenham sido realizados em relação a redução do consumo de água nas indústrias do setor, a geração de efluentes e consequentemente a de resíduos, como o lodo provenientes das estações de tratamento são inevitáveis (Barbosa, 2014). Outro problema que advém do crescimento industrial é a inserção e aumento do descarte de metais pesados no ambiente.

A contaminação de corpos hídricos por meio de efluentes industriais contendo metais pesados é um dos problemas ambientais mais significativos devido a sua natureza tóxica e acúmulo desses íons metálicos na cadeia alimentar (Gautam et al., 2014). Quando a concentração destes metais pesados, lançados no meio ambiente por inúmeros processos industriais tais como os 
metalúrgicos, de mineração, de galvanoplastia, de curtimento, os petroquímicos, de fundição, de fabricação de fertilizantes, alcalis, pesticidas, pigmentos, explosivos e outros é maior que os padrões estabelecidos pelos órgãos ambientais competentes, inicia-se o processo de poluição e/ou contaminação do meio, tendo por consequência a alteração das características químicas, físicas e biológicas, conferindo sérios prejuízos ao bem estar dos seres vivos em geral e a saúde humana (Veneu et al., 2013; Gautam et al., 2014).

Existem diversas técnicas de tratamentos convencionais para remoção de metais pesados, dentre estes podemos destacar a precipitação química, troca iônica, separação por membranas, técnica eletroquímicas, oxidação, extração com solvente, flotação e adsorção (Kargi e Cikla, 2007; Veneu et al., 2012). No entanto, as baixas eficiências de remoção destes poluentes em soluções muito diluídas e os elevados custos de alguns destes métodos praticamente impossibilitam as suas aplicações. Nos últimos anos, processos alternativos de tratamento empregando materiais biológicos como microalgas, bactérias, fungos, leveduras e resíduos agroindustriais e industriais têm sido utilizados para remoção de metais pesados (Veneu et al., 2013).

Neste trabalho, foi avaliado o potencial do lodo proveniente de um decantador primário de um sistema de tratamento de efluentes de lodos ativados em uma indústria do segmento de celulose e papel como sorvente para remoção dos íons metálicos $\mathrm{Cu}, \mathrm{Cd}$ e $\mathrm{Cr}$ em escala de bancada. A influência de parâmetros operacionais como pH inicial da solução e concentração inicial de lodo foram investigadas. Análises de Microscopia Eletrônica de Varredura acoplada a Espectroscopia de Energia Dispersa (MEV/EDS) e Espectroscopia no Infravermelho (IV-TF) foram realizadas antes e após os ensaios de sorção para obtenção da estrutura morfologia e os principais elementos contidos no lodo, assim como os principais grupos funcionais envolvidos no processo de sorção.

\section{MATERIAIS E MÉTODOS}

\subsection{Procedência e Obtenção do Lodo}

A amostra de lodo foi obtida na estação de tratamento de efluentes de uma indústria de fabricação de papéis especiais. Uma amostra de aproximadamente 2,0 $\mathrm{kg}$ de lodo foi obtida do decantador primário do sistema de lodos ativados convencional da indústria. Após a coleta, o lodo foi homogeneizado em beckers de 2,0 L, sendo posteriormente levados a uma estufa de secagem modelo Quimis a uma temperatura de $90{ }^{\circ} \mathrm{C}$, por 48 horas. Após a secagem, obtiveram-se tortas do lodo seco que posteriormente foram desagregadas e peneiradas para a obtenção de partículas de tamanho $<100 \mu \mathrm{m}$.

\subsection{Preparo das Soluções Estoques dos Íons Metálicos}

As soluções foram preparadas com água deionizada a partir dos sais de $\mathrm{CuCl}_{2} \cdot 2 \mathrm{H}_{2} \mathrm{O}(99 \%)$, $3 \mathrm{CdSO}_{4} \cdot \mathrm{H}_{2} \mathrm{O}(98 \%)$ e $\mathrm{CrCl}_{3} \cdot 6 \mathrm{H}_{2} \mathrm{O}(97 \%)$ fornecidos pela VETEC. As soluções teste foram preparadas a partir de soluções estoque com concentração de $500 \mathrm{mg} / \mathrm{L}$ adicionando os volumes necessários da solução estoque para a obtenção das concentrações iniciais empregadas nos ensaios de sorção. 


\subsection{Caracterização do Lodo}

A técnica de Espectroscopia no Infravermelho mediante Transformada de Fourier (IV-FT) foi utilizada para avaliar os principais grupos funcionais envolvidos na sorção dos metais pelo lodo. O lodo antes e após o processo de sorção foi analisado utilizando um espectrofotômetro $F T$ IR Nicolet 2000. Para o estudo de IV-FT, 0,01 g de pó de lodo foram encapsulados em 0,1 g de $\mathrm{KBr}$, a fim de preparar as pastilhas translúcidas, obtendo assim, os espectros no comprimento de onda de 4000 a $400 \mathrm{~cm}^{-1}$.

As micrografias foram obtidas em um Microscópio Eletrônico de Varredura (MEV) FEI Quanta 400, equipado com Sistema de Energia Dispersa por espectrometria de raios X (EDS) Bruker Xflash 4030 para avaliar a morfologia do lodo antes e após os ensaios de sorção. Foram utilizadas amostras com aproximadamente $0,1 \mathrm{~g}$ de lodo, previamente metalizadas por meio de precipitação a vácuo de uma película micrométrica de ouro sobre a superfície das mesmas, sendo estas, utilizadas para geração das micrografias. A aquisição e tratamentos dos dados gerados pelo EDS foram feitos por meio do software ESPRIT versão 9.1 da Brucker.

\subsection{Experimentos de Sorção em Batelada}

Todos os ensaios de sorção foram realizados em beckers de $500 \mathrm{~mL}$, empregando um volume de solução metálica de $250 \mathrm{~mL}$ em um Jar Test modelo Q305M3 da Quimis com uma velocidade de rotação de $150 \mathrm{rpm}$, tempo de contato de $60 \mathrm{~min}$ a temperatura ambiente de $25 \pm 2$ ${ }^{\circ} \mathrm{C}$. Após cada ensaio, o lodo era então concentrado por meio de filtração em papel de filtro de 1,2 $\mu \mathrm{m}$, sendo removido para que a solução metálica após o processo de sorção seja coletada e transformada em amostra, sendo estas acidificadas com uma solução de $\mathrm{HNO}_{3}(0,1 \mathrm{M})$ para sua preservação e posterior análise da concentração residual do metal por análise elementar em ICP/OES em um equipamento Perkin Elmer Optima 4300DV. Foram utilizados como controle dos ensaios, brancos contendo água deionizada e lodo, com o objetivo de reduzir os possíveis efeitos de interferentes de matriz.

\section{RESULTADOS E DISCUSSÃO}

\subsection{Efeito do pH}

Dentre os fatores que influenciam o processo de sorção, o $\mathrm{pH}$ da solução desempenha um papel importante e afeta a química da solução de metais e da atividade dos grupos funcionais do sorvente. Foram realizados os ensaios de sorção em diferentes valores de $\mathrm{pH}$ escolhidos dentro da faixa de solubilidade dos metais estudados. A Figuras 1 mostra o efeito do $\mathrm{pH}$ na sorção das espécies de $\mathrm{Cu}, \mathrm{Cd}$ e $\mathrm{Cr}$ pelo lodo.

Na Figura 1 pode ser observado que o $\mathrm{pH}$ exerce um papel importante no percentual de remoção dos íons metálicos no processo de sorção. Em pH 2, o percentual de remoção dos íons $\mathrm{Cu}$ eram de aproximadamente 16,3\%, a medida que o pH é incrementado, a remoção eleva-se para valores na faixa de 55\%, na faixa de $\mathrm{pH}$ de 3 a 4 . Os melhores percentuais de remoção foram observados no valor de $\mathrm{pH}$ de 5, correspondendo a 69,4\%. Pode-se observar um comportamento semelhante para os íons $\mathrm{Cd}$ e $\mathrm{Cr}$, em $\mathrm{pH} 2$, foram obtidos os resultados menos expressivos, 
correspondendo a $23,4 \%$ e $11,5 \%$, respectivamente. A medida que os valores de $\mathrm{pH}$ foram aumentando, as remoções foram também incrementadas, atingindo uma espécie de platô em $\mathrm{pH} 4$ para o $\mathrm{Cd}$ e pH 3 para o $\mathrm{Cr}$, obtendo remoções de 96,4\% e 99\%, respectivamente. A partir destes valores de $\mathrm{pH}$, as remoções mantêm-se constantes para os demais.

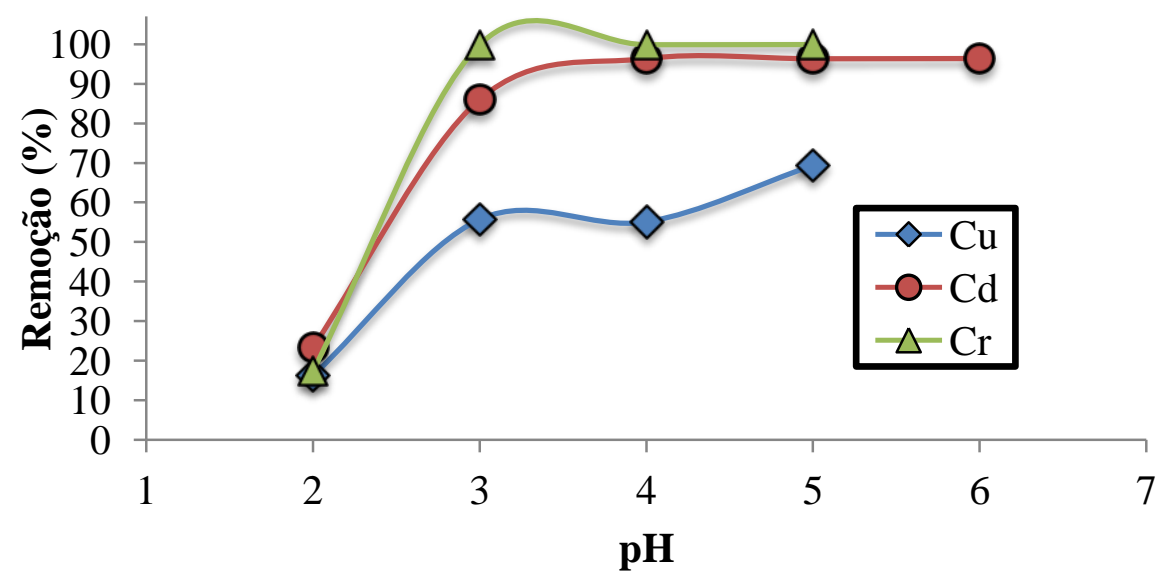

Figura 1 - Efeito do pH na sorção de $\mathrm{Cu}, \mathrm{Cd}$ e $\mathrm{Cr}$ por lodo (concentração inicial de metal: 50 $\mathrm{mg} / \mathrm{L}$; concentração de biomassa: $1,0 \mathrm{~g} / \mathrm{L}$; velocidade de rotação: $150 \mathrm{rpm}$; temperatura: $25{ }^{\circ} \mathrm{C}$; e tempo de contato: $60 \mathrm{~min}$ ).

Bux e Kasan (1994), descreveram que os lodos ativados possuem carga negativa superficial, o que facilita a sua ligação com cátions metálicos positivos. No entanto, dependendo do $\mathrm{pH}$ da solução, os cátions metálicos $\mathrm{Me}^{+}$e outras espécies carregadas positivamente como prótons $\mathrm{H}^{+} \mathrm{e}$ outros da solução podem competir pelos sítios ativos da biomassa. Hammaini et al. (2007), em seus estudos empregando lodo ativado como sorvente dos íons $\mathrm{Cd}^{2+}, \mathrm{Cu}^{2+}, \mathrm{Pb}^{2+}, \mathrm{Ni}^{2+}$ e $\mathrm{Zn}^{2+}$ observou que em valores muito baixos de $\mathrm{pH}(\mathrm{pH} 1$ a 2) a captação dos metais foram insignificantes, aumentando com o incremento do $\mathrm{pH}$ para a faixa de 4 a 5 , acima destes valores, não foram observadas melhorias na capacidade de sorção, determinando como pH ideal para sorção os valores de 4 para o $\mathrm{Cd}^{2+}$ e $\mathrm{Cu}^{2+}, 5$ para o $\mathrm{Pb}^{2+}$ e Ni${ }^{2+}$ e 6 para $\mathrm{Zn}^{2+}$.

Segundo Selatnia et al. (2004), em valores de pH muito baixo (com uma concentração elevada de prótons) ligantes da parede celular seriam estritamente associados ao $\mathrm{H}_{3} \mathrm{O}^{+}$, e o acesso de íons metálicos aos ligantes seria restringida, como resultado de forças repulsivas, a medida que o pH é elevado, mais ligantes com carga negativa estariam exposto com o subsequente aumento dos sítios de atração para os íons metálicos carregados positivamente.

\subsection{Efeito da Concentração Inicial de Lodo}

O efeito da concentração inicial de lodo na remoção das espécies de $\mathrm{Cu}, \mathrm{Cd}$ e $\mathrm{Cr}$ é mostrada na Figura 2. Em muitos casos, doses menores de sorvente causam uma menor eficiência na porcentagem de remoção (Asku e Çağatay, 2006; Vijayaraghavan et al., 2006). Na Figura 2, observa-se que o efeito da concentração inicial de lodo na sorção de $\mathrm{Cu}, \mathrm{Cd}$ e $\mathrm{Cr}$ seguem uma tendência similar, com o aumento na concentração inicial de lodo a remoção foi incrementada. 


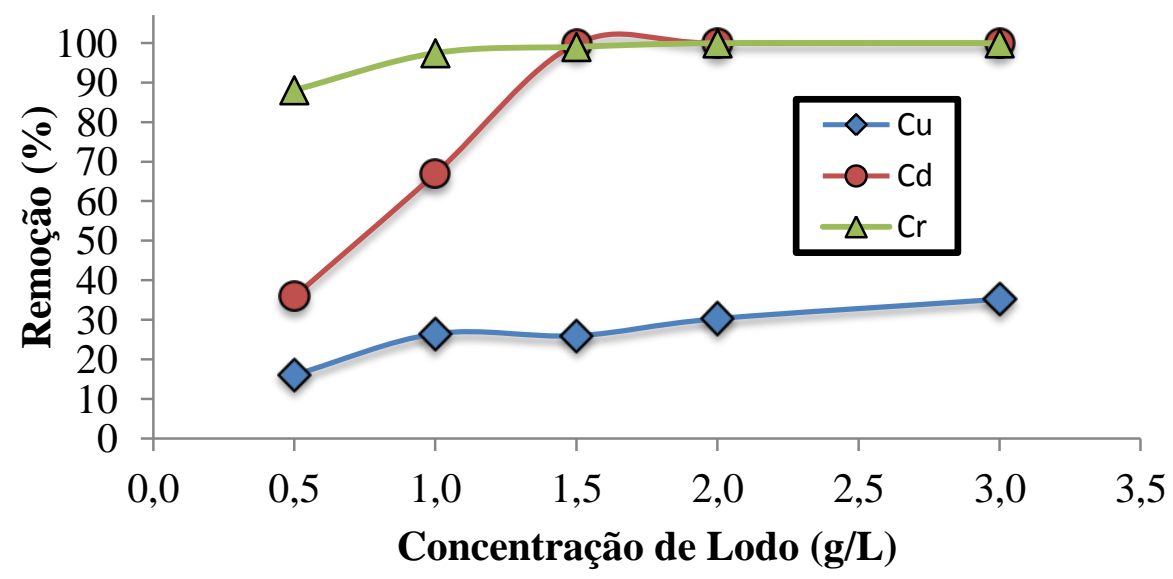

Figura 2 - Efeito da concentração inicial de lodo na sorção de $\mathrm{Cu}, \mathrm{Cd}$ e $\mathrm{Cr}$ (concentração inicial de metal: $100 \mathrm{mg} / \mathrm{L}$; pH: 5,0; velocidade de rotação: $150 \mathrm{rpm}$; temperatura: $25{ }^{\circ} \mathrm{C}$; e tempo de contato: $60 \mathrm{~min})$.

Pode-se observar que a remoção de $\mathrm{Cu}$ na concentração de $0,5 \mathrm{~g} / \mathrm{L}$ é a menor (16\%), nas concentrações de 1,0 e 1,5 g/L as remoções são praticamente idênticas (26\%), o melhor resultado é observado na concentração de $3,0 \mathrm{~g} / \mathrm{L}$, correspondendo a $35 \%$. O mesmo comportamento também é observado para os íons $\mathrm{Cd}$ e $\mathrm{Cr}$, a remoção é baixa na concentração de $0,5 \mathrm{~g} / \mathrm{L}$ e aumenta consideravelmente a medida que a concentração de lodo é incrementada, atingindo um platô em uma concentração de 1,5 g/L, correspondendo a valores de aproximadamente $99 \%$ para as espécies de $\mathrm{Cd}$ e $\mathrm{Cr}$.

Al-Qodah (2006), relatou o efeito da concentração inicial de um lodo ativado proveniente de um laticínio na remoção de íons Cd. Neste estudo, a eficiência de remoção aumentava com o aumento da concentração de lodo, sugerindo que este fenômeno era resultado do aumento dos sítios de ligação para os íons Cd. Segundo Kargi e Cikla (2007), utilizando um resíduo de lodo em pó para remoção de Zn em um reator de fluxo contínuo (CSTR), observou que a remoção de Zn aumentou após $15 \mathrm{~h}$ de operação quando a concentração de lodo passou de 1 para $6 \mathrm{~g} / \mathrm{L}$, diminuindo o residual de Zn na saída do tratamento de 144 para $60 \mathrm{mg} / \mathrm{L}$. Em concentrações muito baixas de sorvente (1 a 2 g/L), a remoção de $\mathrm{Zn}$ foi limitada pela disponibilidade dos sítios de ligação do lodo, no entanto, quando a concentração em solução do sorvente foi elevada para valores de 5 a $6 \mathrm{~g} / \mathrm{L}$, os sítios de ligação sobre as superfícies do lodo excederam o número de íons de $\mathrm{Zn}$ em solução e grandes frações destes íons foram sorvidos sobre a superfície do lodo, resultando em uma baixa concentração residual de $\mathrm{Zn}$.

\subsection{Espectroscopia no Infravermelho}

Buscando identificar os grupos funcionais que seriam os possíveis responsáveis pela sorção dos metais antes e após os ensaios, realizou-se a análise de infravermelho (IV-FT) obtendo os

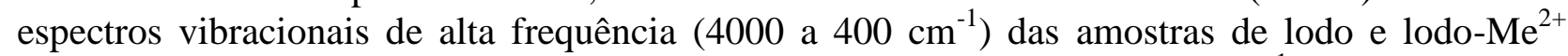
(Figura 3). Todos os espectros mostram forte absorção na região de $3400 \mathrm{~cm}^{-1}$ que podem estar associados ao estiramento de grupos $\mathrm{O}-\mathrm{H}$ característicos de compostos poliméricos. Alterações na 
região de $2900 \mathrm{~cm}^{-1}$ estão relacionado a vibração assimétrica de $\mathrm{CH}_{2}$ e de $2500 \mathrm{~cm}^{-1}$ a vibração S$\mathrm{H}$ de mercaptanos . Uma leve interação em $1600 \mathrm{~cm}^{-1}$ sugere uma fraca interação com grupos C-O$\mathrm{O}, \mathrm{C}=\mathrm{O}$ e $\mathrm{C}-\mathrm{N}$ de ligações peptídicas de proteínas.

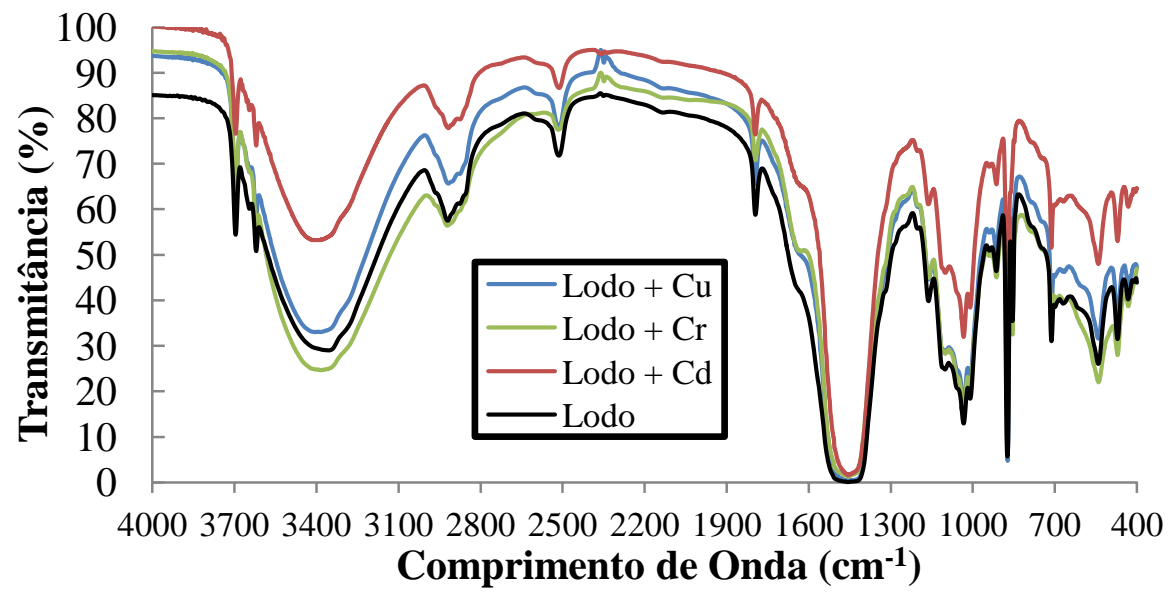

Figura 3 - Espectros na região do infravermelho para as amostras de lodo antes e após os ensaios de sorção com os íons $\mathrm{Cu}, \mathrm{Cd}$ e $\mathrm{Cr}$.

As interações observadas na região de $1450 \mathrm{~cm}^{-1}$ a $1508 \mathrm{~cm}^{-1}$ correspondem ao estiramento das ligações $\mathrm{C}=\mathrm{O}$ provavelmente do $\mathrm{CaCO}_{3}$ com os íons metálicos ou ligações $\mathrm{O}-\mathrm{H}$ de hidróxidos. Os picos observados na região entre 1130 a $1000 \mathrm{~cm}^{-1}$ podem corresponder a ligações Si-O, relacionados às vibrações de ligações $\mathrm{C}-\mathrm{O}-\mathrm{C}$ e $\mathrm{O}-\mathrm{H}$ de polissacarídeos, o que parece ser o mais coerente uma vez que a matriz utilizada é rica em fibras de celulose como pode ser observado nas micrografias de MEV (Figura 4a).

\subsection{Microscopia Eletrônica de Varredura / Espectrometria de Energia Dispersiva de Raios-X}

Dentre as técnicas que podem ser utilizadas na investigação da estrutura e morfologia de partículas está a microscopia eletrônica de varredura (MEV). Porém, embora esta técnica seja provida de uma elevada resolução espacial, possui limitações quanto às informações sobre a composição química da amostra em estudo. Desta forma, a MEV é utilizada em acoplamento com a espectrometria de energia dispersiva de raios-X (EDS), que possui a capacidade de melhorar significativamente os resultados obtidos pela MEV mostrando aspectos qualitativos da composição química da partícula sob investigação (Warwick e Tonner, 1997).

Na Figura 4a, podemos observar a micrografia do lodo antes do processo de sorção obtida pelo MEV que mostra uma grande presença de fibras de celulose e cargas minerais utilizadas no processo de fabricação de papel. No espectro de EDS (Figura 4b), podemos observar sinais dos elementos $\mathrm{Ca}, \mathrm{Al}$, Si e $\mathrm{O}$ que sugerem a presença de carbonato de cálcio $\left(\mathrm{CaCO}_{3}\right)$, sulfato de alumínio $\mathrm{Al}_{2}\left(\mathrm{SO}_{4}\right)_{3}$ e talco $\left(3 \mathrm{MgO} \cdot 4 \mathrm{SiO}_{2} \cdot \mathrm{H}_{2} \mathrm{O}\right)$, produto utilizados para garantir algumas propriedades ao papel. A confirmação da sorção dos íons metálicos $\mathrm{Cu}, \mathrm{Cd}$ e $\mathrm{Cr}$ no lodo após o processo de sorção pode ser observada nos espectros de EDS como mostra as Figuras 5a, b e c, respectivamente. 

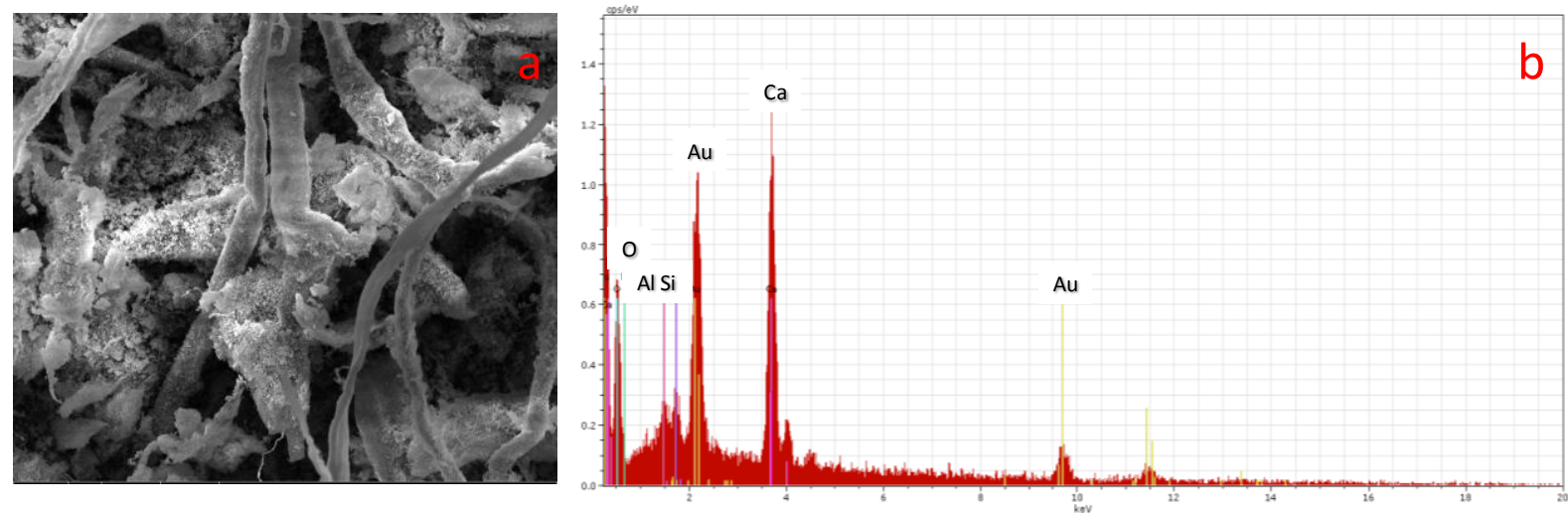

Figura 4 - (a) Micrografia do lodo obtida por MEV (Magnificação - 1000 x); (b) Espectro de EDS do lodo.

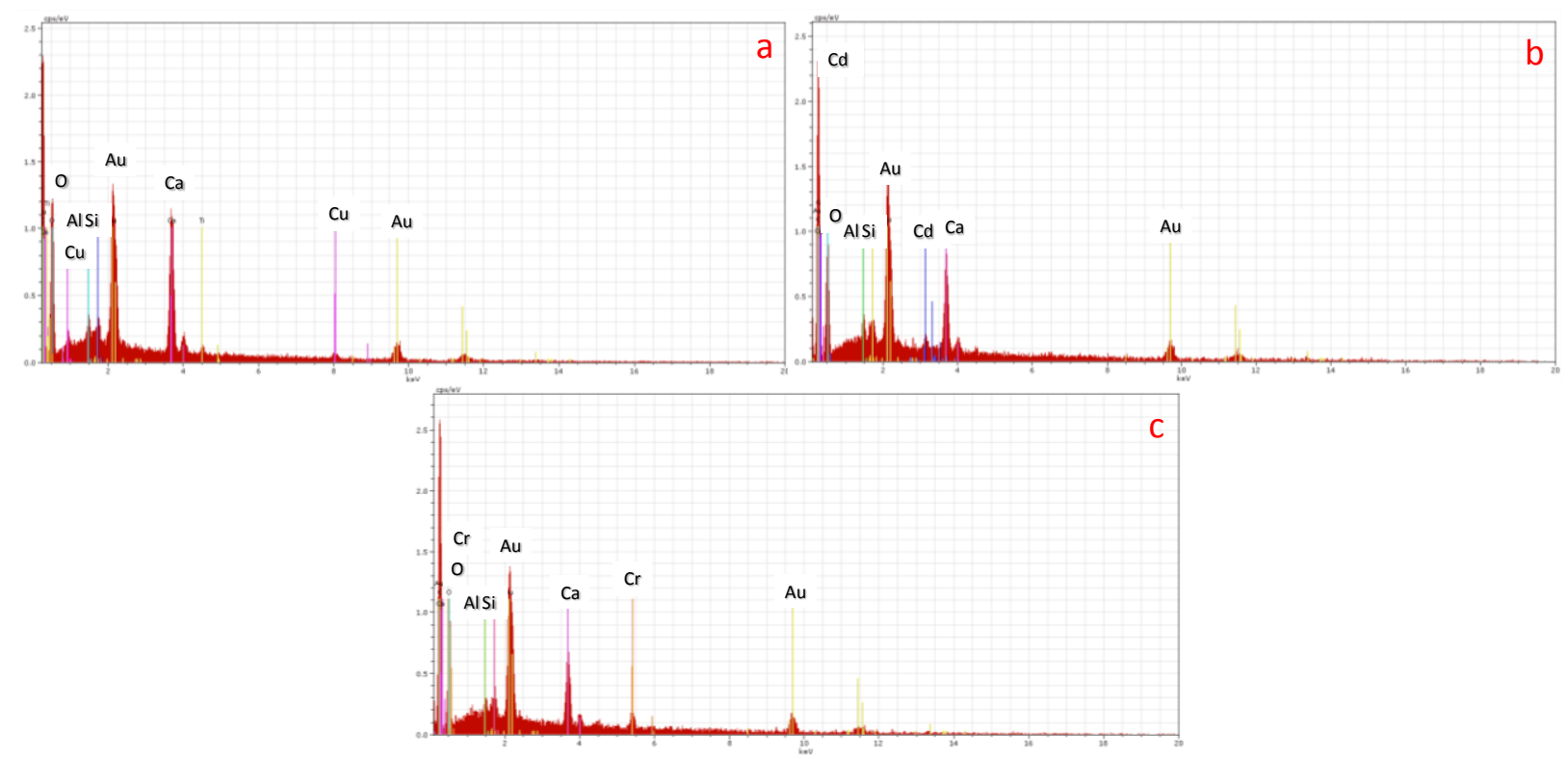

Figura 5 - Espectros de EDS após o processo de sorção: (a) Lodo-Cu; (b) Lodo-Cd; (c) Lodo-Cr.

\section{CONCLUSÃO}

Diante dos resultados obtidos, apresentados e discutidos no presente trabalho, pode-se concluir que a remoção das espécies $\mathrm{Cu}, \mathrm{Cd}$ e $\mathrm{Cr}$ pelo lodo é fortemente influenciada pelo $\mathrm{pH}$ do meio, obtendo-se a maior eficiência em $\mathrm{pH}$ 5,0 para o $\mathrm{Cu}$ e $\mathrm{Cd}$, correspondendo aos valores de $69,4 \%$ e $96,4 \%$, respectivamente, e $\mathrm{pH} 4,0$ para o $\mathrm{Cr}$, correspondendo ao valor de 99,9\%. A remoção dos íons $\mathrm{Cu}, \mathrm{Cd}$ e $\mathrm{Cr}$ sofreram uma relevante influência pela concentração inicial de lodo, sendo os melhores resultados obtidos com 3,0 g/L, correspondendo aos valores de 35,2\%, $99,9 \%$ e $99,8 \%$, respectivamente. As interações entre os íons metálicos e os grupos funcionais da biomassa foram confirmadas pelas análises de IV-FT e MEV/EDS. Os grupos funcionais envolvidos na sorção das espécies metálicas incluíram os grupos carboxila, amina, mercaptanos e hidroxila. 


\section{REFERÊNCIAS BIBLIOGRÁFICAS}

Bracelpa. Associação Brasileira de Celulose e Papel, 2014. Disponível em: http://www.bracelpa.org.br Acesso em: 18 de fevereiro de 2014.

Barbosa, B.B. Utilização de lodo de estação de tratamento de efluentes. Dissertação de Mestrado, Mestrado Profissional em Ciências Ambientais - Universidade Severino Sombra, XX p., 2014.

Gautam, R.K.; Mudhoo, A.; Lofrano, G.; Chattopadhyaya, M.C. Biomass-derived biosorbents for metal ions sequestration: Adsorbent modification and activation methods and adsorbent regeneration. Journal of Environmental Chemical Engineering, v. 2, p. 239-259, 2014.

Veneu, D.M.; Torem, M.L.; Pino, G.A.H. Fundamental aspects of copper and zinc removal from aqueous solutions using a Streptomyces lunalinharesii strain. Minerals Engineering, v. 48, p. 44$50,2013$.

Kargi, F.; Cikla, S. Determination of model parameters for zinc(II) ion biosorption onto powdered waste sludge (PWS) in a fed-batch system. Journal of Environmental Management, v. 85, p. 883890, 2007.

Veneu, D.M.; Pino, G.A.H.; Torem, M.L.; Saint'Pierre, T.D. Biosorptive removal of cadmium from aqueous solutions using a Streptomyces lunalinharesii strain. Minerals Engineering, v. 29, p. 112-120, 2012.

Bux, F.; Kasan, H.C. Comparison of selected methods for relative assessment of sulfate charge on waste sludge biomass. Water SA, v. 20, p. 73-76, 1994.

Hammaini, A.; González, F.; Ballester, A.; Blázquez, M.L.; Muñoz, J.A. Biosorption of heavy metals by activated sludge and their desorption characteristics. Journal of Environmental Management, v. 84, p. 419-426, 2007.

Setatnia, A.; Madami, A.; Bakhti, M.Z.; Keryous, L.; Mansouri, Y.; Yous, R. Biosorption of $\mathrm{Ni}^{2+}$ from aqueous solution by a $\mathrm{NaOH}$-treated bacterial dead Streptomices rimosus biomass. Minerals Engineering, v. 17, p. 903-911, 2004.

Aksu, Z.; Çağatay, S.S. Investigation of biosorption of Gemazol Turquise Blue-G reactive dye by dried Rhizopus arrhizus in batch and continuous systems, Separation Purification Technology, v. 48, p. 24-35, 2006.

Vijayaraghavan, K.; Palanivelu, K.; Velan, M. Biosorption of copper(II) and cobalt(II) from aqueous solutions by crab shell particles, Bioresource Technology, 97: 1411-1419, 2006.

Al-Qodah, Z. Biosorption of heavy metal ions from aqueous solutions by activated sludge. Desalination, v. 196, p. 164-176, 2006.

Warwick, T.; Ade, H.; Hitchcock, H.; Padmore, H.; Rightor, E.; Tonner, B. Soft X-ray spectromicroscopy development for materials science at the advanced light source Journal of Electron Spectroscopy and Related Phenomena, v. 84, p. 85-98, 1997. 